\title{
INTEGRAL FORMULAS AND HYPERSPHERES IN A SIMPLY CONNECTED SPACE FORM
}

\author{
IRL BIVENS
}

\begin{abstract}
Let $M^{n}$ denote a connected compact hypersurface without boundary contained in Euclidean or hyperbolic $n+1$ space or in an open hemisphere of $S^{n+1}$. We show that if two consecutive mean curvatures of $M$ are constant then $M$ is in fact a geodesic sphere. The proof uses the generalized Minkowski integral formulas for a hypersurface of a complete simply connected space form. These Minkowski formulas are derived from an integral formula for submanifolds in which the ambient Riemannian manifold $\bar{M}$ possesses a generalized position vector field; that is a vector field $Y$ whose covariant derivative is at each point a multiple of the identity. In addition we prove that if $\bar{M}$ is complete and connected with the covariant derivative of $Y$ exactly the identity at each point then $\bar{M}$ is isometric to Euclidean space.
\end{abstract}

Let $(M, g)$ denote a connected compact oriented $n$ dimensional Riemannian manifold without boundary with $h: M \rightarrow \bar{M}$ an isometric immersion of $M$ into an oriented $n+p$ dimensional Riemannian manifold $(\bar{M}, \bar{g})$. If $p=1$ we let $\sigma_{k}$ denote the $k$ th mean curvature of $M$. That is $\sigma_{k}$ is equal to the $k$ th elementary symmetric function of the principal curvatures of $M$ divided by $\left(\begin{array}{l}n \\ k\end{array}\right)$.

THEOREM 1. Suppose $p=1$ and assume $\sigma_{k}, \sigma_{k+1}$ are constant for some $k=$ $1,2, \ldots, n-1$.

(a) If $\bar{M}=\mathbf{R}^{n+1}$ with the standard metric then $h$ embeds $M$ as an $n$-sphere in $\mathbf{R}^{n+1}$.

(b) If $\bar{M}=\mathbf{H}^{n+1}$ (hyperbolic $n+1$ space) then $h$ embeds $M$ as a geodesic $n$-sphere in $\mathbf{H}^{n+1}$.

(c) If $\bar{M}=S^{n+1}$ with the standard metric and $h(M)$ is contained in an open hemisphere then $h$ embeds $M$ as an $n$-sphere in $S^{n+1}$.

The additional hypothesis of (c) cannot be omitted since there exist nonspherical compact minimal $\left(\sigma_{1}=0\right)$ hypersurfaces of $S^{n+1}$ with constant scalar curvature (which implies $\sigma_{2}$ is constant).

Part (a) of Theorem 1 was proved by Robert Gardner [2] using the generalized Minkowski integral formulas of Hsiung [4]. Gardner's argument may be applied to prove (b) and (c) once we have the Minkowski formulas for hypersurfaces of $\mathbf{H}^{n+1}$ and $S^{n+1}$ respectively. To describe these integral formulas we need the notion of a

Received by the editors June 28, 1982.

1980 Mathematics Subject Classification. Primary 53C21, 53C25, 53C40; Secondary 53C42.

Key words and phrases. Minkowski integral formulas, totally umbilic submanifold, Codazzi tensor, space form. 
generalized position vector field. Let $\bar{\nabla}$ denote the Riemannian connection of $\bar{M}$ and suppose $Y$ is a vector field on $\bar{M}$.

DEFINITION 1. $Y$ is a generalized position vector field of $\bar{M}$ provided $\bar{\nabla} Y=f I$ where $f$ is a smooth function on $\bar{M}$ and $I$ denotes the identity section of End $(T \bar{M})$.

EXAMPLES. (i) If $\bar{M}=\mathbf{R}^{n+p}, \bar{g}$ is the standard flat metric and $Y=\sum x_{i} \partial / \partial x_{i}$ is the ordinary position vector field then $\bar{\nabla} Y=I$.

(ii) Let $\bar{M}=S^{n+p}$ with $\bar{g}$ the standard metric of constant curvature 1 and let $X$ denote a parallel vector field on $\mathbf{R}^{n+p+1}$. Define a vector field $Y$ on $S^{n+p}$ by letting $Y(x)$ equal the orthogonal projection of $X(x)$ onto $T_{x} S^{n+p}$. Then $\bar{\nabla} Y=(X \cdot N) I$ where $N$ denotes the inward unit normal vector field on $S^{n+p}$.

More generally suppose $X$ is a parallel vector field on a Riemannian manifold $(\hat{M},\langle\rangle$,$) and \bar{M}$ is a totally umbilic submanifold of $\hat{M}$ with $\bar{g}$ the induced metric. If we define a vector field $Y$ on $\bar{M}$ as above then $\bar{\nabla} Y=\langle X, \eta\rangle I$ where $\eta$ denotes the mean curvature normal vector field of $\bar{M}$. In particular if $\bar{M}=\hat{M}$ then $\bar{\nabla} Y=0$.

(iii) Let $\bar{M}=\mathbf{H}^{n+p}=\left\{\left(x_{1}, x_{2}, \ldots, x_{n+p}\right) \in \mathbf{R}^{n+p} \mid x_{n+p}>0\right\}$ with $\bar{g}=$ $\left(1 / x_{n+p}^{2}\right) \sum d x_{i}^{2}$ and $Y=\partial / \partial x_{n+p}$. Then $\bar{\nabla} Y=-I / x_{n+p}$.

(iv) Choose any smooth metric on $S^{n+p-1} \subset \mathbf{R}^{n+p}$. We extend this metric to

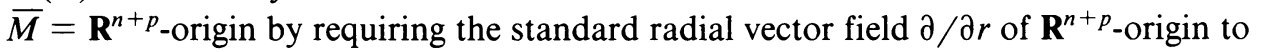
be of unit length and orthogonal to $S^{n+p-1}$ and by also requiring parallel translation along radial directions to be ordinary Euclidean parallel translation. Then for $Y=r \partial / \partial r$ we have $\bar{\nabla} Y=I$. Furthermore with this metric $\bar{M}$ is flat if and only if the original metric gave $S^{n+p-1}$ constant curvature 1 .

The author would like to thank Mike Anderson and Bill Dunbar for suggesting respectively the examples of the generalized position vector fields on $S^{n+p}$ and $\mathbf{H}^{n+p}$.

The second ingredient necessary to describe the integral formulas is the notion of a Codazzi tensor. Suppose $S$ is a tensor of type $(k, k)$ on $M$ which is alternating in the first $k$ and in the last $k$ indices. Then at each point $x \in M, S_{x}$ may be identified with an element of End $\Lambda^{k} T_{x} M$ and we write $S \in \Gamma\left[\right.$ End $\left.\Lambda^{k}(T M)\right]$. Given $S \in$ $\Gamma\left[\right.$ End $\left.\Lambda^{k}(T M)\right]$ and $T \in \Gamma\left[\right.$ End $\left.\Lambda^{j}(T M)\right]$ define $S * T \in \Gamma\left[\right.$ End $\left.\Lambda^{k+j}(T M)\right]$ by wedging together the respective covariant and contravariant components of $S$ and $T$. The multiplication $*$ is associative and commutative. Let $\nabla$ denote the Riemannian connection on the full tensor algebra of $M$.

Definition 2. $S \in \Gamma\left[\right.$ End $\left.\Lambda^{k}(T M)\right]$ is a Codazzi tensor of type $(k, k)$ provided

$$
0=\sum(-1)^{j+1}\left(\nabla_{X_{j}} S\right)\left(X_{1} \wedge X_{2} \wedge \cdots \wedge X_{j-1} \wedge X_{j+1} \wedge \cdots \wedge X_{k+1}\right)
$$

for all $C^{\infty}$ vector fields $X_{1}, X_{2}, \ldots, X_{k+1}$.

It is routine to show that if $S$ and $T$ are Codazzi tensors of types $(k, k)$ and $(j, j)$ respectively then $S * T$ is a Codazzi tensor of type $(k+j, k+j)$.

EXAMPLES. (a) The identity section $I_{k}$ of End $\Lambda^{k}(T M)$ is trivially a Codazzi tensor of type $(k, k)$ since $\nabla I_{k}=0$.

(b) Suppose $p=1, \bar{M}$ has constant sectional curvature and $N$ is a unit normal vector field on $M$. The shape operator $T$ of $M$ is a tensor of type $(1,1)$ on $M$ defined 
by $T(X)=-\bar{\nabla}_{X} N$ where $X$ denotes a tangent vector to $M$. Since $\bar{M}$ has constant sectional curvature the Codazzi equations for $M$ imply $T$ is a Codazzi tensor of type $(1,1)$. As a consequence $S=T^{k}=T * T * \cdots * T$ ( $k$ times) is a Codazzi tensor of type $(k, k)$ and trace $S=k !\left(\begin{array}{l}n \\ k\end{array}\right) \sigma_{k}$ (the factor of $k$ ! occurs because of our particular normalization of wedge products).

(c) The curvature operators $R_{2 k} \in \Gamma\left[\right.$ End $\left.\Lambda^{2 k}(T M)\right]$ are defined by

$$
\left\langle R_{2}(X \wedge Y), Z \wedge W\right\rangle=\langle R(X, Y) Z, W\rangle
$$

and

$$
R_{2 k}=R_{2} * R_{2} * \cdots * R_{2} \quad(k \text { times })
$$

where $R$ denotes the curvature tensor of $M$ and $\langle$,$\rangle denotes the extension of g$ to the exterior algebra of $M$. The second Bianchi identity implies $R_{2}$ is a Codazzi tensor of type $(2,2)$ and thus $R_{2 k}$ is a Codazzi tensor of type $(2 k, 2 k)$.

(d) Suppose $\mathscr{F}$ is a codimension $k$ distribution on $M$ and $\mathcal{H}$ is the distribution orthogonal to $\mathscr{F}$. Let $P$ denote orthogonal projection onto $\mathcal{K}$. Then $P$ is a Codazzi tensor of type $(1,1)$ if and only if $\mathscr{F}$ and $\mathcal{H}$ are parallel distributions (that is invariant under parallel translation along any curve). $S=P^{k}$ is a Codazzi tensor of type $(k, k)$ if and only if $\mathcal{F}$ is integrable with totally geodesic leaves and $\mathcal{H}$ is "minimal" in the sense that $P\left(\sum \nabla_{e_{i}} e_{i}\right)=\sum \nabla_{e_{i}} e_{i}$ whenever $e_{1}, e_{2}, \ldots, e_{k}$ is a local orthonormal frame field for $\mathcal{H}$. For example if $M=S^{3}$ and $\mathscr{F}$ is the tangent space to the fibres of the Hopf fibration $S^{3} \rightarrow S^{2}$ then $S=P^{2}$ is a Codazzi tensor of type $(2,2)$ even though $P$ itself is not a Codazzi tensor.

Assume $Y$ is a generalized position vector field on $\bar{M}$ and define a tensor $A$ of type $(1,1)$ on $M$ by the equation $g(A(X), Z)=\bar{g}(\mathrm{II}(X, Z), Y)$ where II denotes the second fundamental form of $M$.

THeOREM 2. If $S$ is a Codazzi tensor of type $(k, k)$ on $M$ then

$$
\int_{M}(n-k) f \operatorname{trace} S+\operatorname{trace} S * A d V=0 .
$$

Proof. Assume first that $S$ is a Codazzi tensor of type $(n-1, n-1)$. Define an $n-1$ form $\alpha$ on $M$ by $\left.\alpha=Y^{\tan }\right\lrcorner d V$ where $Y^{\tan }$ denotes the orthogonal projection of $Y$ onto $T M$. It is routine to show that if $X$ is any smooth vector field on $M$ then $\left.\nabla_{X} \alpha=[f X+A(X)]\right\lrcorner d V$. Let $e_{1}, e_{2}, \ldots, e_{n}$ denote a local oriented orthonormal frame field for $M$ with $E_{j}=e_{1} \wedge e_{2} \wedge \cdots \wedge e_{j-1} \wedge e_{j+1} \wedge \cdots \wedge e_{n}$. If we consider $\omega=\alpha \circ S$ as an $n-1$ form then

$$
\begin{aligned}
d \omega\left(e_{1}, e_{2}, \ldots, e_{n}\right) & =\sum(-1)^{j+1}\left(\nabla_{e_{j}} \omega\right) E_{j} \\
& =\sum(-1)^{j+1}\left(\nabla_{e_{j}} \alpha\right) S\left(E_{j}\right)+\alpha\left[\sum(-1)^{j+1}\left(\nabla_{e_{j}} S\right) E_{j}\right] \\
& \left.=\sum(-1)^{j+1}\left[f e_{j}+A\left(e_{j}\right)\right]\right\lrcorner d V S\left(E_{j}\right) \\
& =f \operatorname{trace} S+\operatorname{trace} S * A .
\end{aligned}
$$

Since $\int_{M} d \omega=0$ the result follows in this case. 
Suppose now that $S$ is a Codazzi tensor of type $(k, k)$ and apply the above result to $S * I_{n-k-1}$. Then

$$
0=\int_{M} f \operatorname{trace} S * I_{n-k-1}+\operatorname{trace} S * A * I_{n-k-1} d V
$$

which implies

$$
0=\int_{M}(n-k) f \operatorname{trace} S+\operatorname{trace} S * A d V .
$$

Theorem 2 was proved in [1] for the case $\bar{M}=\mathbf{R}^{n+p}$ with the standard metric and with $Y$ the ordinary position vector field. By making different choices of $\bar{M}, Y$ and $S$ we get a number of interesting integral formulas. For example if $S=I$ Theorem 2 becomes

$$
0=\int_{M} f+\bar{g}(\eta, Y) d V
$$

where $\eta$ denotes the mean curvature normal vector field of $M$. As a consequence $M$ cannot be minimally immersed into the open subset of $\bar{M}$ defined by $f \neq 0$. In particular we immediately obtain the (well-known) result that there are no compact minimal submanifolds of Euclidean or hyperbolic space. Similarly there exist no compact minimal submanifolds contained in an open hemisphere of $S^{n+p}$. As another application if we let $\bar{M}=\mathbf{R}^{n+p}$ with the standard metric and let $Y=\partial / \partial x_{i}$, $i=1,2, \ldots, n+p$, then Theorem 2 implies

$$
\int_{M} \eta d V=\overrightarrow{0}
$$

Finally we derive the generalized Minkowski formulas for a compact hypersurface in a simply connected space form.

Corollary 3 (Minkowski formulas). Assume $p=1, N$ is a unit normal vector field on $M$ and $\rho=\bar{g}(N, Y)$.

(a) If $\bar{M}=\mathbf{R}^{n+1}$ with the standard metric and $Y=\Sigma x_{i} \partial / \partial x_{i}$ then

$$
\int_{M} \sigma_{k}+\rho \sigma_{k+1} d V=0, \quad k=0,1, \ldots, n-1,
$$

where $\sigma_{0} \equiv 1$.

(b) If $\bar{M}=S^{n+1}$ and $Y$ equals the orthogonal projection of $-\partial / \partial x_{1}$ onto $S^{n+1}$ then

$$
\int_{M} x_{1} \sigma_{k}+\rho \sigma_{k+1} d V=0, \quad k=0,1, \ldots, n-1 .
$$

(c) If $\bar{M}=\mathbf{H}^{n+1}$ and $Y=\partial / \partial x_{n+1}$ then

$$
\int_{M}-x_{n+1}^{-1} \sigma_{k}+\rho \sigma_{k+1} d V=0, \quad k=0,1, \ldots, n-1 .
$$

Proof. For each choice of $\bar{M}$ the shape operator $T$ is a Codazzi tensor of type $(1,1)$ and $A=\rho T$. Let $S=T^{k}, k=0,1, \ldots, n-1\left(T^{0} \equiv I\right)$ and apply Theorem 2 . 
The formulas of part (a) were proved by Hsiung in [4]. Using the above integral formulas and Gardner's argument [2] we now prove Theorem 1.

Proof of Theorem 1 . Since $M$ is compact there exists a point on $M$ at which all principal curvatures are positive. Thus $\sigma_{k} \neq 0, \sigma_{k+1} \neq 0$ and it follows that $\sigma_{k}^{2}-$ $\sigma_{k-1} \sigma_{k+1} \geqslant 0$ with equality if and only if $M$ is totally umbilic [3]. Our hypotheses imply that for appropriate $f$ and $\rho$

$$
\int_{M} f \sigma_{j}+\rho \sigma_{j+1} d V=0, \quad j=0,1, \ldots, n-1,
$$

where $f \neq 0$ on $M$. Consequently

$$
0=\sigma_{k} \int_{M} f \sigma_{k}+\rho \sigma_{k+1} d V=\int_{M} f \sigma_{k}^{2}+\rho \sigma_{k} \sigma_{k+1} d V
$$

and

$$
0=\sigma_{k+1} \int_{M} f \sigma_{k-1}+\rho \sigma_{k} d V=\int_{M} f \sigma_{k-1} \sigma_{k+1}+\rho \sigma_{k} \sigma_{k+1} d V
$$

Subtracting the second equation from the first yields

$$
0=\int_{M} f\left(\sigma_{k}^{2}-\sigma_{k-1} \sigma_{k+1}\right) d V
$$

Since $f \neq 0$ on $M, \sigma_{k}^{2}-\sigma_{k-1} \sigma_{k+1} \equiv 0$ and $M$ is totally umbilic. For each choice of $\bar{M}$ the only compact totally umbilic hypersurfaces are the geodesic spheres and Theorem 1 follows.

In light of Theorem 2 it is natural to ask what Riemannian manifolds possess a generalized position vector field such that $f \equiv 1$. Examples (i) and (iv) give two such manifolds, the first of which is complete and the second of which is noncomplete. In fact, we have the following result.

THEOREM 4. Suppose $(\bar{M}, \bar{g})$ is a complete connected Riemannian manifold and $Y$ is a vector field on $\bar{M}$ such that $\bar{\nabla} Y=I$. Then $\bar{M}$ is isometric to Euclidean space and under this isometry $Y$ corresponds to the ordinary position vector field of Euclidean space.

Proof. The unit vector field $E=Y /\|Y\|$ is defined on the open subset $\{q \in \bar{M} \mid$ $Y(q) \neq 0\}$. Since $\bar{\nabla}_{E} E=[(1-E[\|Y\|] /\|Y\|)] E$ must be orthogonal to $E$, it follows that $E[\|Y\|]=1$ and that the integral curves of $E$ are geodesics of $\bar{M}$. Thus if $\gamma_{q}(t)$ denotes the unique geodesic such that $\gamma_{q}^{\prime}(\|Y(q)\|)=E(q)$ then $Y\left(\gamma_{q}(t)\right)=t \gamma_{g}^{\prime}(t)$ for $t \geqslant 0$ since $\bar{M}$ is complete. In particular $Y$ has at least one zero on $\vec{M}$. Since Hess $\|Y\|^{2}=2 \bar{g}$ the function $\|Y\|^{2}$ is strictly convex and therefore $Y$ has exactly one zero $p \in \bar{M}$. By considering a normal neighborhood of $p$ we conclude that if $\gamma(t)$ is a unit speed geodesic with $\gamma(0)=p$ then $Y(\gamma(t))=t \gamma^{\prime}(t), t \geqslant 0$. This implies the exponential mapping exp: $T_{p} \bar{M} \rightarrow \bar{M}$ is one to one. It is onto by the completeness of $\bar{M}$. Furthermore since

$$
\bar{R}(X, Z) Y=-\bar{\nabla}_{X} \bar{\nabla}_{Z} Y+\bar{\nabla}_{Z} \bar{\nabla}_{X} Y+\nabla_{[X, Z]} Y=-\bar{\nabla}_{X} Z+\bar{\nabla}_{Z} X+[X, Z]=0
$$


it follows from the Jacobi equation that the exponential mapping is nonsingular. Thus exp: $T_{p} \bar{M} \rightarrow \bar{M}$ is a diffeomorphism and we make the identification $\left(T_{p} \bar{M}, \exp ^{*} \bar{g}\right)=(\bar{M}, \bar{g})$.

Let $\left(r, \theta_{1}, \theta_{2}, \ldots, \theta_{N}\right), N+1=\operatorname{dim} \bar{M}$, denote a system of spherical coordinates on the inner product space $\left(T_{p} \bar{M}, \bar{g}_{p}\right)$. Then $E=\partial / \partial r, Y=r \partial / \partial r$ with

$$
\bar{\nabla}_{\partial / \partial r} \frac{\partial}{\partial r}=\bar{\nabla}_{E} E=0
$$

and with

$$
\begin{aligned}
\bar{\nabla}_{\partial / \partial r} \frac{1}{r} \frac{\partial}{\partial \theta_{i}} & =-\frac{1}{r^{2}} \frac{\partial}{\partial \theta_{i}}+\frac{1}{r} \bar{\nabla}_{\partial / \partial r} \frac{\partial}{\partial \theta_{i}}=-\frac{1}{r^{2}} \frac{\partial}{\partial \theta_{i}}+\frac{1}{r} \bar{\nabla}_{\partial / \partial \theta_{i}} \frac{\partial}{\partial r} \\
& =-\frac{1}{r^{2}} \frac{\partial}{\partial \theta_{i}}+\frac{1}{r^{2}} \bar{\nabla}_{\partial / \partial \theta_{i}} Y=-\frac{1}{r^{2}} \frac{\partial}{\partial \theta_{i}}+\frac{1}{r^{2}} \frac{\partial}{\partial \theta_{i}}=0 .
\end{aligned}
$$

Therefore the vector fields $\partial / \partial r,(1 / r) \partial / \partial \theta_{i}, i=1,2, \ldots, N$, are parallel in the radial directions with respect to the metric $\exp ^{*} \bar{g}$. On the other hand these vector fields are clearly parallel in the radial directions with respect to the flat metric $\hat{g}$ induced on $T_{p} \bar{M}$ via $\bar{g}_{p}$ and the natural identification $T_{q}\left(T_{p} \bar{M}\right) \approx T_{p} \bar{M}$. Since $\hat{g}=\exp ^{*} \bar{g}$ at the origin of $T_{p} \bar{M}$ it follows that $\hat{g}=\exp ^{*} \bar{g}$ and $\bar{M}$ is isometric to Euclidean $N+1$ space.

Theorem 4 is equivalent to a result of Tashiro [5] which we now de $\lrcorner$ cribe. Tashiro shows that if $\phi$ is a smooth function on a complete Riemannian manifold $(\bar{M}, \bar{g})$ such that Hess $\phi=2 c \bar{g}, 0 \neq c \in \mathbf{R}$, then $\bar{M}$ is isometric to Euclidean space. Since $\bar{\nabla} Y=I$ implies Hess $\|Y\|^{2}=2 \bar{g}$, Theorem 4 follows as a corollary. Conversely, given such a function $\phi$ then the vector field $Y=1 / 2 c \operatorname{grad} \phi$ satisfies $\bar{\nabla} Y=I$ and Tashiro's result may be derived from Theorem 4 .

The author wishes to thank Robert Reilly for pointing out that parts (c) of Theorem 1 and (b) of Corollary 3 are contained in his paper, Extrinsic rigidity theorems for compact submanifolds of the sphere, J. Differential Geom. 4 (1970), 487-497.

\section{REFERENCES}

1. I. Bivens, Codazzi tensors and reducible submanifolds, Trans. Amer. Math. Soc. 268 (1981), 231-245.

2. R. Gardner, The Dirichlet integral in differential geometry, Global Analysis, S. S. Chern and S. Smale, editors, Proc. Sympos. Pure Math, vol. 15, Amer. Math. Soc., Providence, R. I., 1970, pp. 231-237.

3. G. H. Hardy, J. E. Littlewood and G. Pólya, Inequalities, Cambridge Univ. Press, Cambridge, 1934.

4. C. C. Hsiung, Some integral formulas for closed hypersurfaces, Math. Scand. 2 (1954), 286-294.

5. Y. Tashiro, Complete Riemannian manifolds and some vector fields, Trans. Amer. Math. Soc. 117 (1965), 251-275.

Department of Mathematics, Rice University, Houston, Texas 77251

Current address: Department of Mathematics, Davidson College, Davidson, North Carolina 28036 